\title{
A CHARACTERIZATION AND SUM DECOMPOSITION FOR OPERATOR IDEALS
}

\author{
BY \\ ANDREAS BLASS ${ }^{1}$ AND GARY WEISS
}

\begin{abstract}
Let $L(H)$ be the ring of bounded operators on a separable Hilbert space. Assuming the continuum hypothesis, we prove that in $L(H)$ every two-sided ideal that contains an operator of infinite rank is the sum of two smaller two-sided ideals. The proof involves a new combinatorial description of ideals of $L(H)$. This description is also used to deduce some related results about decompositions of ideals. Finally, we discuss the possibility of proving our main theorem under weaker assumptions than the continuum hypothesis and the impossibility of proving it without the axiom of choice.
\end{abstract}

1. Introduction and notational conventions. Let $H$ be a separable, infinite-dimensional, complex Hilbert space, and let $L(H)$ be the ring of bounded linear operators on $H$. Assuming the continuum hypothesis, we shall prove that every two-sided ideal $I$ of $L(H)$ that properly includes the ideal of finite-rank operators can be decomposed as the sum $I=J_{1}+J_{2}$ of two-sided ideals $J_{i}$ properly included in $I$. The question whether such a decomposition exists for the ideal $K(H)$ of compact operators was raised by Brown, Pearcy and Salinas [1]. We shall also show that the decomposition of $K(H)$ is necessarily nonconstructive, in the sense that there need not be any definable ideals $\subsetneq K(H)$ whose sum is $K(H)$.

Our proof is in two parts. The first part develops a new characterization of ideals in terms of sequences of natural numbers and thereby reduces the problem to a combinatorial one. The second part uses the continuum hypothesis to solve this combinatorial problem. The two parts are presented in $\$ \S 2$ and 3, respectively. $\$ 4$ contains some additional comments.

Before starting the proof, we adopt some notational conventions. By the sum of two sequences of real numbers, we mean the sequence obtained by adding corresponding terms; similarly, one sequence is $\leqslant$ another if every

Received by the editors May 5, 1976 and, in revised form, July 20, 1977.

AMS (MOS) subject classifications (1970). Primary 46K05, 47B05; Secondary 02K20, 02K25.

Key words and phrases. Hilbert space, operator ideals, compact operators, continuum hypothesis, axiom of choice, Calkin ideal sets.

${ }^{1}$ Partially supported by NSF Grant GP43760. 
term of the former is $\leqslant$ the corresponding term of the latter. The sum of two sets consists of all possible sums of two members, one from each set. (We have already used this convention in writing $J_{1}+J_{2}$ above.) A subset $A$ of the set $\mathbf{N}$ of natural numbers is said to be almost included in another such set $B$ if $A-B$ is finite; a sequence of such sets is said to be almost decreasing if each term is almost included in every earlier term. By an ideal we always mean a two-sided proper ideal of $L(H)$. We write $K(H)$ and $F(H)$ for the ideals of compact operators and of finite-rank operators, respectively. $(K(H)$ is the largest ideal, and $F(H)$ is the smallest nonzero ideal.) Finally $i$ is a variable taking only the values 1 and 2 . With these conventions, our main theorem takes the following form.

Theorem 1. Assume the continuum hypothesis. For every ideal $I \supsetneqq F(H)$, there exist two ideals $J_{i} \subsetneq I$ such that $J_{1}+J_{2}=I$.

2. The characterization of ideals. We begin by recalling the description of ideals given by von Neumann and Calkin [2]. To each ideal $I$, associate the Calkin ideal set Calk(I), consisting of all the sequences obtainable by taking any nonnegative selfadjoint operator in $I$ and listing (in any order) its eigenvalues, repeating multiple eigenvalues according to their multiplicities. Since $I \subseteq K(H)$, Calk $(I)$ is a subclass of the class $c_{0}^{+}$of sequences of nonnegative real numbers converging to zero. Furthermore, Calk $(I)$ is easily seen to be closed under the relations permute, decrease, and add defined as follows:

Permute: A sequence $x$ yields a sequence $y$ by permute if the same positive numbers occur in $x$ and $y$ with the same multiplicites.

Decrease: $x$ yields $y$ by decrease if $y \leqslant x$.

Add: $x$ and $y$ yield $x+y$ by add.

It is shown in [2] that every nonempty subset of $c_{0}^{+}$that is closed under permute, decrease, and add is Calk $(I)$ for a unique ideal $I$. An easy corollary, which we shall need later, is that the lattice 9 of ideals is distributive. (Our formulation differs from Calkin's in that permute allows insertion or deletion of zeros. Calkin shows that this is redundant, but it will be convenient for our purposes.) In this characterization of Calkin ideal sets, add can be replaced by the following two relations.

Double: $x$ yields $y$ by double if $y=2 x$, i.e., $y_{n}=2 x_{n}$ for all $n$.

Mesh: $x$ and $y$ yield $z$ by mesh if the multiplicity of occurence in $z$ of each real number is the sum of its multiplicities in $x$ and $y$.

Indeed, double is obviously a special case of add, while mesh is obtainable from permute and add (use permute to insert infinitely many zeros). Conversely, the sum of two sequences is obtainable by double and decrease from their (term-by-term) maximum, which is a subsequence of a sequence given 
by mesh. One can extract the subsequence by using decrease to replace the unwanted terms by zeros and then using permute to remove these zeros. Thus, the Calkin ideal sets are precisely the nonempty subsets of $c_{0}^{+}$closed under permute, decrease, double, and mesh.

Let $b$ be the subset of $c_{0}^{+}$consisting of sequences all of whose nonzero terms are of the form $1 / 2^{n}$ with $n \in \mathbf{N}$. For each $x \in c_{0}^{+}$there exists $y \in b$ such that $y_{n} \leqslant x_{n} \leqslant 2 y_{n}$ for all but finitely many $n$ (the exceptional values of $n$ are those for which $x_{n}>2$ ). Then any nonzero Calkin ideal set contains $x$ if and only if it contains $y$. Thus, a Calkin ideal set is uniquely determined by its intersection with $b$. Furthermore, a nonempty subset of $b$ is $b \cap \operatorname{Calk}(I)$ for some ideal $I$ if and only if it is closed under permute, decrease (restricted to $b$ ), mesh, and a modified form of double in which only terms $<1$ are doubled while ones are left unchanged (so that the result still lies in $b$ ).

A sequence $x \in b$ can be specified uniquely, modulo permute, by giving the multiplicity of each power of $\frac{1}{2}$ in $x$. It is more convenient for our purposes, however, to use cumulative multiplicities; we define $x^{*}(n)$ to be the total number of occurrences of $1,1 / 2, \ldots, 1 / 2^{n}$ in $x$. Thus, for each $x \in b, x^{*}$ belongs to the set $s$ of nondecreasing functions from $\mathbf{N}$ to $\mathbf{N}$. An ideal $I$ is completely determined by $b \cap \operatorname{Calk}(I)$, which is in turn completely determined by the subset

$$
I^{*}=\left\{x^{*} \mid x \in b \cap \operatorname{Calk}(I)\right\}
$$

of $s$. By translating the characterization of sets of the form $b \cap \operatorname{Calk}(I)$, we find that sets of the form $I^{*}$ are characterized, among all subsets of $s$, by closure under decrease (restricted to $s$ ), add, and shift, where we define

Shift: $f$ yields $g$ by shift if $g(n)=f(n+1)$ for all $n$.

We denote the lattice of these sets by $\mathcal{S}$.

It is obvious that the correspondences between ideals, their Calkin ideal sets, their intersections with $b$, and finally their *-transforms all preserve inclusion. Thus, we have the first part of the following result.

THEOREM 2. The correspondence $I \mapsto I^{*}$ is an isomorphism from the lattice 9 of ideals to the lattice $\mathcal{S}$ of subsets of $s$ closed under decrease, add, and shift. In both of these lattices, meet is set-theoretic intersection and join is sum.

The proof of the last sentence of the theorem is trivial except, possibly, the verification that, if two subsets of $s$ are closed under decrease, then so is their sum. This amounts to checking that if $f \leqslant g_{1}+g_{2}$ then $f=h_{1}+h_{2}$ for some $h_{i} \leqslant g_{i}$ (where $f, g_{i}, h_{i}$ all belong to $s$ ); the required functions $h_{i}$ are easily defined by induction. Once $h_{i}(n)$ have been defined for a particular $n$, the requirements for $h_{i}(n+1)$ are $h_{i}(n) \leqslant h_{i}(n+1) \leqslant g_{i}(n+1)$ and $h_{1}(n+1)+$ $h_{2}(n+1)=f(n+1)$; these requirements can be satisfied because $h_{1}(n)+$ $h_{2}(n)=f(n) \leqslant f(n+1) \leqslant g_{1}(n+1)+g_{2}(n+1)$. 
Theorem 2 immediately implies the following corollary, which reduces the proof of Theorem 1 to a combinatorial problem about $s$.

Corollary. An ideal $I$ is the sum of two ideals $J_{i} \subsetneq I$ if and only if $I^{*}$ is the sum of two sets $S_{i} \subsetneq I^{*}$ in $\delta$.

For future reference, we record here that the ideals $F(H)$ and $K(H)$ correspond to $F(H)^{*}$, consisting of all bounded functions in $s$, and $K(H)^{*}=$ $s$.

The first part of the proof of Theorem 1, introducing the combinatorial characterization of ideals, is complete. Before proceeding with the second part ( $\$ 3)$, we present some other results obtainable by combining Theorem 2 with some classical facts about $s$. The facts we have in mind are contained in the following lemma, which is due to Hausdorff [3].

LemMA. Let $f_{k}(k=1,2, \ldots)$ be countably many functions in s. Then there exists $f \in s$ such that, for each $k$, the inequality $f_{k}(n) \leqslant f(n)$ holds for all but finitely many $n$. Furthermore, if each $f_{k}$ is unbounded, then there exists an unbounded $g \in s$ such that, for each $k, g(n) \leqslant f_{k}(n)$ holds for all but finitely many $n$.

Proof. Set $f(n)=\max \left\{f_{k}(n) \mid k \leqslant n\right\}$. Set $g(n)=\max \{l \mid$ for all $k \leqslant l$, $\left.f_{k}(n) \geqslant l\right\}$.

The following corollary slightly strengthens a result of Salinas [8].

Corollary. $K(H)$ is not the union of countably many ideals $I_{k} \subsetneq K(H)$.

Proof. For each $k$, let $f_{k} \in s-I_{k}^{*}$. By the lemma, let $f \in s$ be such that $f_{k} \leqslant f$ almost everywhere (i.e., for all but finitely many values). Then $f$ is in no $I_{k}^{*}$, and it follows that a nonnegative selfadjoint compact operator with eigenvalues $1 / 2^{n+1}$ with multiplicity $f(n+1)-f(n)$ is in no $I_{k}$.

The next corollary shows that the decomposition in Theorem 1 cannot be very simple.

COROLlary. If $K(H)=I_{1}+I_{2}$ with $I_{i} \subsetneq K(H)$, then neither of the ideals $I_{i}$ is countably generated.

Proof. Suppose $I_{1}$ were countably generated. Then $I_{1}^{*}$ would also be countably generated, say by $f_{1}, f_{2}, \ldots$ We may suppose, without loss of generality, that all of the functions obtainable from the $f_{k}$ 's by add and shift are already among the $f_{k}$ 's. Thus

$$
\begin{aligned}
s & =K(H)^{*}=I_{1}^{*}+I_{2}^{*} \\
& =\left\{g \in s \mid \text { for some } h \in I_{2}^{*} \text { and some } k, g \leqslant f_{k}+h\right\} .
\end{aligned}
$$

(It is easy to see the last equality by checking that the right side is in $\delta$ and recalling that, in $\delta,+$ is join.) 
By the lemma, let $f \in s$ be such that $f_{k} \leqslant f$ almost everywhere. For every function $g \in s$, we have $f+g \in s$, so, for some $h \in I_{2}^{*}$ and some $k$, $f+g \leqslant f_{k}+h$. The choice of $f$ then implies that $g \leqslant h$ almost everywhere, so $g \in I_{2}^{*}$. But $g$ was an arbitrary element of $s$, so $s=I_{2}^{*}$ and therefore $K(H)=I_{2}$, a contradiction.

3. The combinatorial construction. We now return to the proof of Theorem 1. Our goal is to find two sets $S_{i} \in \mathcal{S}$ whose sum is a prescribed $S \in \mathcal{S}$, with $S \supsetneqq F(H)^{*}$, i.e., with $S$ containing some unbounded function. From here on, we assume the continuum hypothesis.

The first (and main) step in the construction of the $S_{i}$ is to construct (quite independently of the prescribed $S$ ) certain almost decreasing sequences of subsets of $\mathbf{N}$. We begin with the observation that, if $\left(B_{\xi}\right)$ is an almost decreasing sequence of infinite sets, indexed by a countable well-ordered set, then there is an infinite set $B$ almost included in all the $B_{\xi}$ 's. Indeed, this is trivial if the given sequence is empty (use $\mathbf{N}$ as $B$ ) or has a last term (use that term as $B$ ). Otherwise, we can extract a cofinal subsequence that can be re-indexed by natural numbers, so we need only consider the case where the given sequence is $\left(B_{n}\right)$ indexed by $\mathbf{N}$. In this case, we define $B$ to consist of one element from $B_{0}$, a different element from $B_{0} \cap B_{1}$, yet another element from $B_{0} \cap B_{1} \cap B_{2}$, etc. Such elements can be found because $B_{0} \cap \cdots \cap$ $B_{n}$ almost includes $B_{n}$ and is therefore infinite. It is obvious that $B$ is almost included in each $B_{n}$.

Having made this preliminary observation (which, like the lemma above, goes back to [3]), we now proceed to construct two almost decreasing sequences, $\left(B_{\alpha}^{1}\right)$ and $\left(B_{\alpha}^{2}\right)$, of infinite subsets of $N$, each indexed by the set of all countable ordinal numbers, and having the following property. For any $f \in s$, there is an index $\alpha$ such that, for all $n \in \mathbf{N}$,

$$
\max \left\{\operatorname{next}\left(B_{\alpha}^{1}, n\right), \operatorname{next}\left(B_{\alpha}^{2}, n\right)\right\} \geqslant f(n),
$$

where we use the notation $\operatorname{next}(B, n)$ for the smallest number in $B$ that is $\geqslant n$.

Since the set $s$ has the cardinality of the continuum and the set of countable ordinal numbers has cardinality $\boldsymbol{\kappa}_{1}$, the continuum hypothesis asserts that there is a bijection between these two sets. Fix such a bijection. We shall define the sets $B_{\alpha}^{i}$ by transfinite induction on $\alpha$, in such a way that (*) holds whenever $f$ and $\alpha$ correspond in our fixed bijection. For any countable ordinal number $\alpha$, the $\alpha$ th step of the induction is as follows. Suppose $B_{\xi}^{i}$ has been defined for all $\xi<\alpha$ (and for both values of $i$ ), and suppose $f$ is the function in $s$ that corresponds to $\alpha$. We must find two infinite sets, $B_{\alpha}^{1}$ and $B_{\alpha}^{2}$, such that, first, (*) holds, and, second, $B_{\alpha}^{i}$ is almost included in $B_{\xi}^{i}$ for all $\xi<\alpha$. By our preliminary observation, we can satisfy the second 
requirement; there are infinite sets $A^{i}$ almost included in $B_{\xi}^{i}$ for all $\xi<\alpha$. By taking the $B_{\alpha}^{i}$ to be infinite subsets of $A^{i}$, we automatically satisfy the second requirement, so we direct our efforts toward the first, making (*) hold. We define an increasing sequence

$$
a_{0}^{1}<a_{0}^{2}<a_{1}^{1}<a_{1}^{2}<a_{2}^{1}<a_{2}^{2}<\ldots
$$

of natural numbers, with $a_{k}^{i} \in A^{i}$, such that $a_{k}^{2}>f(n)$ for all $n<a_{k}^{1}$ and such that $a_{k+1}^{1}>f(n)$ for all $n \leqslant a_{k}^{2}$. Since the $A^{i}$ 's are infinite, there is no difficulty in inductively choosing the $a_{k}^{i}$,s. We set $B_{\alpha}^{i}=\left\{a_{k}^{i} \mid k \in \mathbf{N}\right\}$; clearly this is an infinite subset of $A^{i}$. To verify (*), consider any $n \in \mathbf{N}$. If $a_{k}^{1}<n<a_{k}^{2}$ for some $k$, then $\operatorname{next}\left(B_{\alpha}^{1}, n\right)=a_{k+1}^{1}>f(n)$ by our choice of $a_{k+1}^{1}$. Otherwise, $a_{k-1}^{2}<n \leqslant a_{k}^{1}$ for some $k$ (or simply $n \leqslant a_{0}^{1}$ if $k=0$ ), and $\operatorname{next}\left(B_{\alpha}^{2}, n\right)=a_{k}^{2}$ $>f(n)$ by our choice of $a_{k}^{2}$. In either case, (*) holds. This completes the construction of the sequences $\left(B_{\alpha}^{i}\right)$.

Temporarily fix an arbitrary unbounded function $h \in s$. (Eventually, we will select a particular $h$, depending on the prescribed $S \in \mathcal{\delta}$ that we want to decompose, but for the moment the choice of $h$ is immaterial. Some of what follows may seem more natural if the reader pretends that $h$ is the identity function.) We say that a function $f \in s$ is linearly bounded on a set $B \subseteq \mathbf{N}$ if there are constants $a$ and $b$ such that $f(n) \leqslant a h(n)+b$ for all $n \in B$. Let $S_{i}^{\prime}$ ( $i=1$ or 2 ) be the set of all $f \in s$ that are linearly bounded on at least one of the sets $B_{\alpha}^{i}$ defined above. Note that such an $f$ is also linearly bounded on all later terms $B_{\beta}^{i}(\beta>\alpha)$ of the sequence, as these are almost included in $B_{\alpha}^{i}$. It follows that $S_{i}^{\prime}$ is closed under add as well as decrease. It might not, however, be closed under shift. We define $S_{i}$ to be the closure of $S_{i}^{\prime}$ under shift; this is obviously closed under decrease and is easily seen to be closed under add as well. (The verification uses the fact that every $f \in s$ is nondecreasing, so its $n$-fold shift is $\leqslant$ its $m$-fold shift if $n \leqslant m$.) Thus, $S_{1}$ and $S_{2}$ belong to $\delta$.

We show next that $S_{1}+S_{2}=s$. Consider an arbitrary $g \in s$. Since $h$ is unbounded, we can find $f \in s$ such that $h(f(n)) \geqslant g(n)$ for all $n$. By construction of the sequences $\left(B_{\alpha}^{i}\right)$, we can find an $\alpha$ such that (*) holds for $\alpha$ and $f$. Define

$$
g_{i}(n)=h\left(\operatorname{next}\left(B_{\alpha}^{i}, n\right)\right) .
$$

These two functions $g_{i}$ belong to the corresponding $S_{i}$, for $g_{i}$ is linearly bounded on $B_{\alpha}^{i}$ (being equal to $h$ there). And, because of (*) and the monotonicity of $h$,

$$
\begin{aligned}
g_{1}(n)+g_{2}(n) & \geqslant \max \left\{g_{1}(n), g_{2}(n)\right\} \\
& =h\left(\max \left\{\operatorname{next}\left(B_{\alpha}^{1}, n\right), \operatorname{next}\left(B_{\alpha}^{2}, n\right)\right\}\right) \\
& \geqslant h(f(n)) \geqslant g(n) .
\end{aligned}
$$

Therefore, $g \in S_{1}+S_{2}$. 
For any prescribed $S \in \mathcal{S}$, we have, by distributivity of $\mathcal{S}$,

$$
\left(S \cap S_{1}\right)+\left(S \cap S_{2}\right)=S \cap\left(S_{1}+S_{2}\right)=S \cap s=S .
$$

To complete the proof of Theorem 1, we must still show that, if $S$ contains an unbounded function $g$, then $h$ can be so chosen that neither $S_{i}$ includes $S$ (so $S \cap S_{i} \subsetneq S$ ). In fact, we shall find $h$ such that $g$ belongs to neither $S_{i}$, because $g$ and all the functions from which $g$ is obtainable by shift will be linearly bounded only on finite sets. Thus, we seek an unbounded $h \in s$ such that, for any $a, b, c$, the inequality

$$
g(n-c) \leqslant a h(n)+b
$$

holds for only finitely many values of $n$. In other words, we need an unbounded $h \in s$ such that, for each $a, b, c$,

$$
h(n) \leqslant[g(n-c) / a+b / a]
$$

for all but finitely many $n$. But such an $h$ exists by the second part of Hausdorff's Lemma above, so the proof of Theorem 1 is complete.

\section{Additional remarks.}

4.1. Theorem 1 can also be proved by directly constructing the sets $S_{i} \subsetneq S$ such that $S_{1}+S_{2}=S$. The construction is an inductive one, of length $\aleph_{1}$. At each step of the construction, new functions are put into $S_{1}$ and $S_{2}$ to guarantee that some $f \in S$ belongs to $S_{1}+S_{2}$. The continuum hypothesis is needed to insure that each $f \in S$ can be considered at some stage. The construction is so arranged that a particular unbounded $g \in S$ is never put into either $S_{i}$, so $S_{i} \subset S$. The appropriate functions to put into $S_{i}$ at each stage are given by the following "splitting lemma".

LemMa. Let $S_{1}$ and $S_{2}$ be countably generated members of $\mathcal{S}$, let $g_{i} \notin S_{i}$, $g_{i} \in s$, and let $f \in s$ be arbitrary. Then $f$ can be written as $f_{1}+f_{2}$, where $f_{i} \in s$, and where the sets $S_{i}^{\prime} \in \mathcal{S}$ generated by $S_{i} \cup\left\{f_{i}\right\}$ do not contain the corresponding $g_{i}$.

This lemma is perhaps most easily proved by means of a Baire category argument which establishes that "most" decompositions $f_{1}+f_{2}$ of $f$ (with $f_{i} \in s$ ) have the required property. A similar lemma can be proved (with somewhat more work) for the characteristic sets introduced by Salinas [7], and Theorem 1 can also be obtained in this way.

4.2. Theorem 2 can be rather neatly reformulated as follows. Define a binary relation $\leqslant$ on $s$ by

$f \preccurlyeq g$ iff there exist natural numbers $a$ and $b$ such that $f(n) \leqslant a g(n+b)$ for all $n$.

This relation is obviously reflexive and transitive. We write $[f]$ for the 
equivalence class of $f$ with respect to the associated equivalence relation and $l$ for the set of equivalence classes. With the partial ordering induced by $\preccurlyeq, l$ is a distributive lattice in which

$$
\begin{aligned}
& {[f] \wedge[g]=[\min (f, g)] \text { and }} \\
& {[f] \vee[g]=[\max (f, g)]=[f+g] .}
\end{aligned}
$$

It is easy to verify that a subset $S$ of $s$ belongs to $\delta$ if and only if (1) it is closed under add and (2) $f \leqslant g \in S$ implies $f \in S$. Therefore, the sets $S \in \mathcal{S}$ correspond, via the projection $s \rightarrow l$, to the ideals of $l$. Theorem 2 thus asserts that the lattice of ideals (of the ring $L(H)$ ) is isomorphic to the lattice of ideals of the lattice $l$.

4.3. An obvious question about Theorem 1 is whether the continuum hypothesis is really needed. We have been unable to eliminate it entirely from the argument, but we can get by with certain weaker hypotheses. Readers familiar with Martin's axiom [6] will have no difficulty carrying out essentially the same argument (or the argument outlined in 4.1) on the basis of that assumption. The weakest hypothesis from which we have deduced the conclusion of Theorem 1 is

There exist two nonprincipal ultrafilters $D_{i}$ on $\mathbf{N}$ such that $f\left(D_{1}\right) \neq f\left(D_{2}\right)$ for all finite-to-one functions $f: \mathbf{N} \rightarrow \mathbf{N}$.

The proof involves showing that two such ultrafilters have the property (*) required of the sets $\left\{B_{\alpha}^{i} \mid \alpha\right.$ countable $\}$ in $\$ 3$.

The hypothesis (**) is true if, for example, there exist two nonisomorphic rare ultrafilters (take these as the $D_{i}$ ) and also if there exists a rare nonRamsey ultrafilter (take it as $D_{1}$ and any proper image of it as $D_{2}$ ). It is perhaps worth noting in this connection that Kunen's model [5] (generated by $\aleph_{2}$ random reals over a model of the continuum hypothesis), in which there are no Ramsey ultrafilters, nevertheless satisfies (**) because it has plenty of rare ultrafilters (measure algebra extensions preserve rarity). It seems possible that one could prove (**) (without any special assumptions), perhaps by an adaptation of Kunen's techniques in [4], but we have been unable to do this.

4.4. Although the continuum hypothesis might be eliminable from Theorem 1 , the axiom of choice is not. Furthermore, even if the axiom of choice and the (generalized) continuum hypothesis are assumed, there might not exist any definable ideals $I_{i} \subsetneq K(H)$ such that $I_{1}+I_{2}=K(H)$, not even if arbitrary real numbers and arbitrary ordinal numbers are allowed as parameters in the definitions of the $I_{i}$. Thus, Theorem 1 itself, not merely its present proof, is highly nonconstructive.

To prove these assertions, we begin by topologizing the set $s$ as a subspace of the product of countably many copies of the discrete space $N$. More explicitly, for every finite nondecreasing sequence $p$ of natural numbers, we 
define

$$
[p]=\{f \in s \mid p \text { is an initial segment of } f\},
$$

and we use the sets $[p]$ as a basis for the topology. (We reserve the letters $p, q$, and $r$ to stand for such sequences.) The space $s$ so defined admits a complete metric $(d(f, g)=1 /(n+1)$ if $n$ is the smallest integer for which $f(n) \neq g(n))$ and therefore satisfies the Baire category theorem.

We shall need the following results, which are proved in [9] (under the assumption that the existence of an inaccessible cardinal is consistent). There is a model of set theory without the axiom of choice, in which the axiom of dependent choice holds, and in which every subset of $s$ has the Baire property (i.e. differs from an open set by a meager set). There is another model of set theory, in which the axiom of choice and the generalized continuum hypothesis hold, and in which all definable subsets of $s$ have the Baire property, even if real numbers and ordinal numbers occur as parameters in the definition. The axiom of dependent choice, mentioned in the first of these results, is a weak form of choice that suffices for the proofs of "nonpathological" results of real analysis, like the Baire category theorem and our Theorem 2, but not for the construction of "pathological" examples, like nonmeasurable sets or sets lacking the Baire property.

In view of these facts, the following proposition suffices to establish our claims about the nonconstructivity of Theorem 1 .

Proposition. If $s=S_{1}+S_{2}$ with $S_{i} \in \mathcal{S}$, and $S_{i} \subsetneq s$, then at least one of the $S_{i}$ lacks the Baire property.

The proof of this proposition involves two lemmas about comeager subsets of $s$, so we begin by discussing such sets. By definition of the topology, every open set in $s$ has the form

$$
[D]=\bigcup_{p \in D}[p]
$$

for some collection $D$ of nondecreasing finite sequences $p .[D]$ is dense in $s$ if and only if every $q$ has an extension $p \in D$. More generally, $[D] \cap[r]$ is dense in the basic open set [ $r$ ] if and only if every extension $q$ of $r$ has an extension $p \in D$; in this case, we say that $D$ is dense beyond $r$. A set $C \subseteq s$ is comeager in $[r]$ if and only if it includes the intersection of countably many sets $\left[D_{n}\right]$ where each $D_{n}$ is dense beyond $r$.

LEMMA A. If $C$ is comeager in a nonempty open subset of $s$, then every function $f \in s$ is eventually majorized by the sum of two elements of $C$.

Proof. Without loss of generality, the nonempty open set in question is $[r]$ and $C$ is $\bigcap_{n}\left[D_{n}\right]$, where each $D_{n}$ is dense beyond $r$. Let an $f \in s$ be given. We shall construct $g_{i} \in C$ such that $f(k) \leqslant g_{1}(k)+g_{2}(k)$ for all but finitely 
many $k$. The construction will proceed in stages; after stage $n$, we shall have defined finite nondecreasing sequences $g_{1}^{n}$ and $g_{2}^{n}$, of equal length, which are to be initial segments of the desired $g_{1}$ and $g_{2}$. We begin by setting $g_{1}^{0}=g_{2}^{0}=$ $r$. At an odd-numbered stage, say $2 n+1$, we properly extend $g_{1}^{2 n}$ to a sequence $g_{1}^{2 n+1} \in D_{n}$. This is possible, as $D_{n}$ is dense beyond $r$ and $g_{1}^{2 n}$ extends $r$. We also extend $g_{2}^{2 n}$ to $g_{2}^{2 n+1}$, of the same length as $g_{1}^{2 n+1}$, using values so large that $g_{1}+g_{2}$ will majorize $f$ on domain $\left(g_{1}^{2 n+1}\right)-\operatorname{domain}\left(g_{1}^{2 n}\right)$. (For example, the new values of $g_{2}$ could be taken to agree with $f$.) Symmetrically, at even-numbered stages, $2 n+2$, extend $g_{2}^{2 n+1}$ to $g_{2}^{2 n+2} \in D_{n}$ and extend $g_{1}^{2 n+1}$ so that $f$ is majorized. The functions $g_{1}$ and $g_{2}$ thus obtained are in $C$, for stage $2 n+i$ guarantees that $g_{i} \in\left[D_{n}\right]$. And $g_{1}(k)+g_{2}(k) \geqslant f(k)$ for all $k$ greater than the length of $r$.

Lemma B. Let $C$ be comeager in s. There exists an $f \in S$ such that, in any decomposition $f=h_{1}+h_{2}$ with $h_{1}, h_{2} \in s$, one of the summands $h_{i}$ majorizes an element of $C$.

Proof. Without loss of generality, $C=\cap_{n}\left[D_{n}\right]$ with $D_{n}$ dense (beyond the empty sequence). If $h \in s$ and $p$ is a finite nondecreasing sequence, we say that $h$ is $(p, n)$-small if $h$ majorizes $p$ but does not majorize any extension of $p$ in $D_{n}$. (To be precise, " $h$ majorizes $p$ " means that the finite initial segment of $h$ having the same length as $p$ majorizes $p$.) If $h$ is not $(p, n)$-small for any $p$ and $n$, then we can construct $g \in C$ with $g \leqslant h$ by inductivity defining initial segments $g_{n} \in D_{n}$ majorized by $h$. The existence of $g_{n+1}$ follows trivially from the facts that $h$ majorizes $g_{n}$ and $h$ is not $\left(g_{n}, n+1\right)$-small. Thus, it suffices to find an $f \in s$ such that, for any quadruple $\left(p_{1}, n_{1}, p_{2}, n_{2}\right)$,

$f$ has no decomposition $f=h_{1}+h_{2}$ in which each $h_{i}$ is $\left(p_{i}, n_{i}\right)$-small.

By the Baire category theorem, it suffices to show that, for each fixed $\left(p_{1}, n_{1}, p_{2}, n_{2}\right)$, the set of $f$ satisfying $(\dagger)$ has dense interior, that is, that every finite nondecreasing sequence $q$ has an extension $q^{\prime}$ such that every $f$ extending $q^{\prime}$ satisfies $(\dagger)$.

Let $p_{i}, n_{i}$, and $q$ be given. We may assume that $q$ and both of the $p_{i}$ have the same length $l$; otherwise, just extend the shorter ones by repeating their last terms. By density, we can find extensions $r_{i} \in D_{n_{i}}$ of $p_{i}$. Let $a_{i}$ be the largest (i.e. last) term in $r_{i}$. Define $q^{\prime}$ to be the extension of $q$, of length $l+1$, having $a_{1}+a_{2}$ as its last term $q^{\prime}(l)$. If $f$ extends $q^{\prime}$ and $f=h_{1}+h_{2}$, then either $h_{1}(l) \geqslant a_{1}$ or $h_{2}(l) \geqslant a_{2}$. As the $h_{i}$ are nondecreasing, it follows, by the choice of $a_{i}$, that, for at least one of the two values of $i, h_{i}$ majorizes $r_{i}$ if it majorizes $p_{i}$. So, for at least one $i, h_{i}$ is not $\left(p_{i}, n_{i}\right)$-small.

We now complete the proof of the proposition. Suppose $s=S_{1}+S_{2}$ with 
$S_{i} \in \mathcal{S}, S_{i} \subsetneq s$. We remark that neither $S_{i}$ is $\{0\}$, so any $f$ that is majorized almost everywhere by an element of $S_{i}$ must itself be in $S_{i}$. This remark, the closure of $S_{i}$ under add, the assumption that $S_{i} \subsetneq s$, and Lemma A immediately imply that neither $S_{i}$ is comeager in any nonempty open set. On the other hand, if both $S_{i}$ were meager, we could apply Lemma B to the complement of their union. Remembering that the $S_{i}$ are closed under decrease, we would obtain an $f \in s$ such that, in any decomposition of $f$, one of the summands is in neither of the $S_{i}$. This would contradict $f \in s=S_{1}+$ $S_{2}$.

Thus, at least one of the $S_{i}$ is neither comeager in a nonempty open set nor meager. It therefore lacks the Baire property.

\section{REFERENCES}

1. A. Brown, C. Pearcy and N. Salinas, Ideals of compact operators on Hilbert space, Michigan Math. J. 18 (1971), 373-384.

2. J. W. Calkin, Two-sided ideals and congruences in the ring of bounded operators on Hilbert space, Ann. of Math. (2) 42 (1941), 839-873.

3. F. Hausdorff, Die Graduierung nach dem Endverlauf, Abh. Sächs. Ges. Wiss. 31 (1909), 295-334.

4. K. Kunen, Ultrafilters and independent sets, Trans. Amer. Math. Soc. 172 (1972), 299-306.

5. _ Some points in $\beta N$, Math. Proc. Cambridge Philos. Soc. 80 (1976), 385-398.

6. D. A. Martin and R. Solovay, Internal Cohen extensions, Ann. Math. Logic 2 (1970), 143-178.

7. D. Morris and N. Salinas, Semiprime ideals and irreducible ideals in the ring of bounded operators on Hilbert space, Indiana Univ. Math. J. 23 (1974), 575-589.

8. N. Salinas, Symmetric norm ideals and relative conjugate ideals, Trans. Amer. Math. Soc. 188 (1974), 213-240.

9. R. Solovay, A model of set theory in which every set of reals is Lebesgue measurable, Ann. of Math. (2) 92 (1970), 1-56.

10. G. Weiss, Commutators and operator ideals, Thesis, Univ. of Michigan, Ann Arbor, Mich., 1975.

Department of Mathematics, University of Michigan, Ann Arbor, Michigan 48109

Department of Mathematics, University of Cincinnati, Cincinnati, Oho 45221 (Current address of Gary Weiss)

Current address (Andreas Blass): Department of Mathematics, University of Wisconsin, Madison, Wisconsin 53706 